\title{
Alexander Klose Im Schatten der Profession: Rechtsberatungsgesetz und Heilpraktikergesetz
}

Im Vergleich zu anderen Reformvorbaben fast geräuschlos hat die Bundesregierung im August letzten Jahres den Entwurf für ein Gesetz über außergerichtliche Rechtsdienstleistungen (RDGE) $)^{1}$ beschlossen, das das aus dem Jahr 1935 stammende Rechtsberatungsgesetz (RBerG) ablösen soll. Die Anwaltschaft hat sich von Beginn an intensiv an der Reformdiskussion beteiligt, ${ }^{2}$ obgleich der RDGE gerade die Zulässigkeit nichtanwaltlicher Rechtsberatung zum Gegenstand hat. Dies nimmt der Beitrag zum Anlass, nach der Bedeutung des Rechtsanwalts für die Tätigkeit des Rechtsberaters zu fragen, und vergleicht dies mit dem Verhältnis von Arzt und Heilpraktiker.

\section{A. Einleitung}

Rechtsberatungsgesetz (RBerG) und Heilpraktikergesetz (HPG) regeln in Deutschland Tätigkeitsfelder, die zugleich von zwei klassischen Professionen für sich in Anspruch genommen werden. So wie die vom RBerG erfasste »Besorgung fremder Rechtsangelegenheiten « den Kern anwaltlicher Tätigkeit darstellt, betrifft die vom HPG geregelte »Ausübung der Heilkunde« die ärztliche Berufsausübung. Adressaten beider Gesetze sind jedoch gerade nicht Ärzte und Rechtsanwälte, sondern Personen, die - ohne Arzt oder Rechtsanwalt zu sein rechtsbesorgend oder heilend tätig werden wollen.

Unterfielen Rechtsbesorgungen und Heilbehandlungen bis zum Ende der Weimarer Republik der Gewerbefreiheit und waren mit wenigen Einschränkungen »Jedermann-Tätigkeiten «, wurden »Kurier- und Rechtsberatungsfreiheit « mit Erlass des RBerG I935 und des HPG I939 aufgehoben. Heute behält das RBerG die Beratung und Vertretung in Rechtsangelegenheiten in einem in Europa wohl einzigartigen Umfang der Anwaltschaft vor, ${ }^{3}$ während das HPG einen Beruf hervorgebracht hat, der mit seiner umfassenden Befugnis zur Heilbehandlung ohne ärztliche Approbation ebenfalls eine Besonderheit im europäischen Rechtskreis darstellt.

Diese Entwicklung erscheint sowohl in ihren Parallelen als auch in ihren Unterschieden erklärungsbedürftig. Wie werden aus nichtprofessionellen Gewerbetreibenden »Kurpfuscher « und » Winkeladvokaten«? Warum darf ein Heilpraktiker heute ohne Medizinstudium operative Eingriffe vornehmen, während ein pensionierter Richter sich das Recht, unentgeltlichen Rechtsrat zu erteilen, vor dem Bundesverfassungsgericht erstreiten muss?

I Gesetzentwurf der Bundesregierung für ein Gesetz zur Neuregelung des Rechtsberatungsrechts v. I.9.2006 (BR-Drs. 623/06).

2 Stellungnahmen und Presseerklärungen der BRAK finden sich unter www.brak.de/seiten/o7.php\#rechtsber (Zugriff: 5.2.2007).

3 Einen Überblick über die Rechtslage in anderen europäischen Staaten bietet die Gesetzesbegründung zum RDGE (BR-Drs. 623/06, S. $52 \mathrm{ff}$.). 
Die Antwort auf diese Fragen sucht dieser Beitrag, vom sog. »machtkritischen« Ansatz 4 (power approach) in der Professionssoziologies inspiriert, bei den Ärzten und Rechtsanwälten. Professionen werden danach als soziale Konstrukte begriffen, deren Entstehung, in Abgrenzung zur strukturfunktionalistischen Auffassung, nicht die »natürliche « Folge gesellschaftlicher Arbeitsteilung, sondern das Ergebnis sozialer Aushandlungsprozesse ist. Ziel der berufspolitischen Strategien von Professionen ist dabei die Monopolisierung von Wissen, das dann gegen Honorar zur Verfügung gestellt werden kann. Dazu ist neben der Beschränkung des Zugangs zur Profession auf Personen, die (selbst)bestimmten Anforderungen genügen, die Kontrolle des jeweiligen Marktes erforderlich. Insbesondere in Deutschland muss dabei die Rolle des Gesetzgebers berücksichtigt werden, der durch Lizenzierung und Zulassungsbeschränkungen erheblichen Anteil an der Entstehung von Exklusivität und Monopolstellung hat. ${ }^{6}$

Wendet man diese Überlegungen auf die vorliegenden Fragen an, so ist zu untersuchen, welchen Einfluss Professionen auf die Regulierung sub- bzw. nichtprofessioneller Tätigkeit haben. Dabei geht es weniger um den (unmittelbaren) Einfluss von Lobbyvertretern auf den Gesetzgeber als um die Frage, wie sich gesellschaftlich vermittelte Vorstellungen von Professionen im Recht niederschlagen.

Im ersten Teil des Beitrags soll anhand der Entstehungsgeschichte von RBerG und HPG gezeigt werden, dass zu diesen Vorstellungen erstens die umfassende Bedürfnisbefriedigung durch die Profession und zweitens die „Gefährlichkeit“ Nichtprofessioneller gehört. Schutzbedürftig sind dabei in erster Linie die »Verbraucher «, aber auch der Staat und die Profession selbst. Ein berechtigtes Interesse an subprofessioneller Tätigkeit besteht nach Auffassung des Gesetzgebers nur dort, wo es (noch) Lücken in der professionellen »Versorgung « gibt.

Im zweiten Teil werden diese Vorstellungen in Anknüpfung an das an der Freien Universität Berlin durchgeführte rechtstatsächliche »Forschungsprojekt Rechtsberatungsgesetz «7 einer näheren Untersuchung unterzogen. Lassen sich die Annahmen des Gesetzgebers empirisch bestätigen oder ist er womöglich zum »Professionalisierungsagenten « geworden, der auch mit dem RDGE - bewusst oder unbewusst - die berufspolitischen Interessen der Rechtsanwälte befördert?

\section{B. Zwischen Gewerbefreibeit und Verbot}

Hatte das sog. »Noth-Gewerbegesetz « des Norddeutschen Bundes vom 8. Juli I $868^{8}$ den »Gewerbebetrieb der Aerzte, Apotheker, Hebammen, Advokaten, Notare, Seeschiffer, Seesteuerleute und Lootsen« noch von der Anordnung der

4 Grundlegend: Johnson, Terence: Professions and Power, Basingstoke/London 1972; Larson, Magali: The Rise of Professionalism. A Sociological Analysis, Berkley/Los Angeles/London 1977; Freidson, Eliot: Professionalism Reborn. Theory, Prophecy and Policy, Cambridge I994.

5 Wurde noch Mitte der 9oer Jahre das Ende des professionssoziologischen Diskurses im deutschsprachigen Raum beklagt, erfuhr die Professionssoziologie in den letzten Jahren eine » Wiederbelebung «, deren erste "Zwischenbilanz« in: Pfadenhauer, Manuela (Hrsg.): Professionelles Handeln, Wiesbaden 2005, gezogen wird. Kritische Anmerkungen zu den »neuen Wegen der Professionsforschung « von Schmeiser, Martin, Soziologie 2006, S. 178-191.

6 Den schwierigen Weg der Ärzte und Rechtsanwälte in den USA zur Professionalisierung ohne staatliche »Hilfe« beschreibt Göbel, Geschichte und Gesellschaft I990, S. 3 I 8-342.

7 Neben der Projekt-Homepage www.rechtsberatungsgesetz.info: Rottleuthner, Hubert/Klose, Alexander: Das Rechtsberatungsgesetz - rechtstatsächlich betrachtet, Gutachten H für den 65. Deutschen Juristentag, München 2004.

8 »Noth-Gewerbegesetz « des Norddeutschen Bundes v. 8.7.1868 (BGBl. des Norddeutschen Bundes, S. 406). 
Gewerbefreiheit ausgenommen, findet sich eine solche Einschränkung in der

binnen Jahresfrist beschlossenen »Gewerbeordnung des Norddeutschen Bundes « nicht mehr.9 Auch für das Rechts- und Medizinalgewerbe gilt damit $\mathbb{I}$ GewO: »Der Betrieb eines Gewerbes ist Jedermann gestattet, soweit nicht durch dieses Gesetz Ausnahmen oder Beschränkungen vorgeschrieben oder zugelassen sind.«

Eine »Approbation", also eine von einem Befähigungsnachweis abhängige Genehmigung, soll nach $\ 29$ GewO in Zukunft lediglich Voraussetzung für das Führen des Titels »Arzt « ${ }^{10}$ oder »Apotheker «, für eine staatliche Anerkennung als solcher und für die Übernahme amtlicher Funktionen sein, nicht aber für die Ausübung der Heilkunde.

Damit hat im Bereich des Norddeutschen Bundes und seit I87 I im Deutschen Reich die Liberalisierung der rechtsberatenden und heilenden Berufe ihren Höhepunkt erreicht. Jedermann ist berechtigt, sich als Rechtsberater oder Heiler zu betätigen, und muss dabei keinerlei staatliche Eingriffe befürchten, solange er sich an die allgemeinen Gesetze (insb. Strafgesetz) hält. Schon kurze Zeit später beginnt jedoch der Kampf gegen »Kurpfuscher« und »Winkeladvokaten«, womit jeweils nichtanwaltliche bzw. nichtärztliche Gewerbetreibende mit ganz unterschiedlicher fachlicher Qualifikation bezeichnet werden. Die Heterogenität der Gruppe der nichtärztlichen Heiler zeigt sich in der Gründung eigener Berufsverbände, die die Tätigkeit ihrer Mitglieder regeln und überwachen und zugleich öffentlich vor unzuverlässigen Heilbehandlern warnen. ${ }^{\text {I }}$

Bereits I 4 Jahre später erfährt die Gewerbefreiheit im Bereich des »Rechtsgewerbes« durch die Einführung einer Untersagungsmöglichkeit im Einzelfall eine erste Einschränkung: Nach $\ 35$ GewO ist seit I 883 die gewerbsmäßige Besorgung fremder Rechtsangelegenheiten zu untersagen, »wenn Thatsachen vorliegen, welche die Unzuverlässigkeit des Gewerbetreibenden in Bezug auf diesen Gewerbebetrieb darthun. «² Zudem wird eine behördliche Anzeigepflicht bei Eröffnung des Gewerbes eingeführt. Damit ist zwar für die Aufnahme einer (gewerblichen) Tätigkeit lediglich eine formale Anmeldung erforderlich, und die Behörde lässt den Gewerbetreibenden zunächst einmal gewähren. Das Fehlen fachlicher oder sonstiger Eignung (»Zuverlässigkeit«), kann nun aber zur individuellen Untersagung der Tätigkeit führen.

Begründet wird die Notwendigkeit einer Untersagungsmöglichkeit von der Reichsregierung zunächst mit dem Schutzbedürfnis der Auftraggeber vor unfähigen und unzuverlässigen Gewerbetreibenden. ${ }^{13}$ »Winkeladvokaten, Volksadvokaten und Winkelkonsulenten« würden ihre Klienten durch unredliche und ungeschickte Ausführung der Aufträge schädigen und wüssten sich dabei Vergütungen zu verschaffen, die die Gebühren der Rechtsanwälte nicht selten weit überstiegen. Bemerkenswert ist die durchaus differenzierte Betrachtungsweise: Die geschilderten Missstände bestünden keineswegs überall. Namentlich in Bezirken mit einem höheren Bildungsstand der Bevölkerung würde das Gewerbe ausschließlich oder doch ganz überwiegend von zuverlässigen Personen betrieben. »Unzuverlässige Elemente« könnten hier nicht aufkommen.

\footnotetext{
9 Gewerbeordnung für den Norddeutschen Bund v. 21.6.r 869 (BGBl. des Norddeutschen Bundes, S. 245). I0 \29 GewO nennt: »Wundärzte, Augenärzte, Geburtshelfer, Zahnärzte und Thierärzte«.

I I Gillhausen, Gisbert: Das Berufsrecht der Heilpraktiker, Essen 1953, S. i 8 f.

I 2 Art. 5 des Gesetzes, betreffend Abänderung der Gewerbeordnung v. I.7.I 883 (RGBl. S. I 59).

I 3 Gesetzentwurf zur Änderung der Gewerbeordnung v. I9.4.I 88 I (Aktenstück Nr. 98 zu den Verhandlungen des Reichstages I 88 I, S. 577 ff.).
} 
Daneben wird der Schutz von Gerichten und Behörden als Gesetzeszweck genannt. Neben deren weitgehend nutzloser »Belästigung « sei eine Schädigung ihres Ansehens gerade »in den unteren Volksklassen « zu befürchten, da nichtanwaltliche Gewerbetreibende den unteren Behörden fälschlich Unkenntnis und Ungerechtigkeit unterstellten, um so die Verfahren »bis in die höchsten Instanzen zu treiben. « Damit in Zusammenhang steht die Bekämpfung der Streit- und Prozesssucht, für deren Förderung die Gewerbetreibenden verantwortlich gemacht werden. Sie wüssten die »niedrigsten Leidenschaften « des Publikums auszunutzen, um diese zu »frivolen Prozessen und Denunziationen, zu grundlosen Beschwerden und aussichtlosen Gesuchen « zu verleiten.

Damit sind die von Nichtprofessionellen (vermeintlich) drohenden Gefahren bereits in ihren wesentlichen Ausprägungen benannt. Lediglich der Konkurrenzschutz für die Anwaltschaft findet noch keine Erwähnung. Hier wird vielmehr betont, dass trotz der »schädlichen Auswüchse« durchaus ein Bedürfnis nach nichtanwaltlichen Rechtsberatern bestehe. Der »gemeine Mann« sei nicht in der Lage, sich eines Rechtsanwalts bedienen zu können, da diese »sich mit den geringfügigen Gerichts- und Verwaltungsangelegenheiten « nur ungern befassen würden. Ein vollständiges Verbot sei daher weder möglich noch wünschenswert. ${ }^{\mathrm{I}} 4$

Vor dem Hintergrund der weiteren Rechtsentwicklung interessant ist die Ablehnung der von einigen Behörden geforderten (Wieder)Einführung eines von Zuverlässigkeit und Bedürfnis abhängigen Genehmigungsvorbehalts. Eine solche Genehmigung würde vom Publikum als eine behördliche Garantie für Befähigung und Vertrauenswürdigkeit missverstanden werden mit der Folge, dass sich neben den Rechtsanwälten ein weiterer rechtsberatender Beruf ausbilden könnte, bei dem jedoch keine vergleichbaren Kenntnisse oder Berufspflichten gewährleistet seien.

Die Begründung beruht nach Angaben der Reichsregierung auf Ermittlungen der Behörden in Preußen, wo man etwa 6.600 Rechtsgewerbetreibende gezählt hat, von denen mindestens 2.870 als unzuverlässig eingestuft wurden. Offen bleibt dabei die Frage, was Maßstab für das Verdikt der »Unzuverlässigkeit« war. Die Begründung weist stattdessen an anderer Stelle selbst darauf hin, dass es objektive Anhaltspunkte für die Beurteilung der erforderlichen Befähigung, die je nach Art der geplanten Geschäfte unterschiedlich sei, nicht gebe. Es wird daher zugestanden, dass »dem Ermessen der untersagenden Behörde allerdings ein gewisser Spielraum eingeräumt « werde.

Vor vergleichbaren Eingriffen bleibt das »Medizinalgewerbe« zunächst verschont. Zwar warnt die 1903 gegründete »Deutsche Gesellschaft zur Bekämpfung des Kurpfuschertums « vor unseriösen Heilmethoden (sog. »Geheimmitteln«), sammelt belastendes Material gegen »Kurpfuscher« und erstattet Strafanzeigen gegen sie, ${ }^{15}$ scheitert jedoch 1908/1909 mit dem Versuch, eine dem $\$ 35$ GewO entsprechende Untersagungsmöglichkeit mit Melde-, Buchführungs- und Auskunftspflichten einzuführen. Der Entwurf eines »Gesetzes gegen Mißstände im Heilgewerbe« wurde vom Reichstag an die zuständigen Ausschüsse überwiesen, ohne dass es zu einer Entscheidung in der Sache gekommen wäre. ${ }^{16}$ Auch eine »Lösung « der Kurpfuscherfrage im Rahmen der Strafrechts-

\footnotetext{
I4 In der Reichstagsdebatte des Entwurfs wird dabei der Hoffnung Ausdruck verliehen, dass die »freie Advokatur diesem Mangel an Rechtsanwälten langfristig entgegenwirken und sich das Bedürfnis nach nichtanwaltlichen Beratern entsprechend reduzieren werde. (Protokoll der 35. Sitzung des Reichstages am 28.4. I 88 I, S. 885 ).

Is Freder, Janin, Die Geschichte des Heilpraktikerberufs in Deutschland, Bonn 2003, S. 68.

I6 Freder (Fn. I 5), S. 69 f.
} 
reform durch die Neufassung des Begriffs der »Körperverletzung « wird I 930 vom Reichstag abgelehnt. ${ }^{17}$ Die Kurierfreiheit bleibt damit - abgesehen von Einschränkungen, die sich im Wesentlichen auf die Behandlung ansteckender Krankheiten beziehen ${ }^{18}$ - im Grundsatz bis zum Ende der Weimarer Republik erhalten.

Es bleibt den Nationalsozialisten vorbehalten, die Gewerbefreiheit in beiden Bereichen weitgehend einzuschränken. Mit der Einführung von RBerG ${ }^{19}$ und $\mathrm{HPG}^{20}$ ist die Besorgung fremder Rechtsangelegenheiten sowie die Ausübung der Heilkunde nur noch Personen gestattet, die entweder Inhaber einer entsprechenden Erlaubnis (Heilpraktiker und Rechtsbeistände) oder ausdrücklich vom Verbot ausgenommen sind (wie z.B. Ärzte und Rechtsanwälte). Erklärtes Ziel ist - im bewussten Gegensatz zum »marxistisch-liberalistischen Parteienstaat $\ll^{21}$ die Vereinheitlichung und politische sowie »rassische« Säuberung der Berufslandschaft.

So wird die Erlaubnis nach dem RBerG gem. \$s der Ersten Ausführungsverordnung (I. AVO RBerG) ${ }^{22}$ »Juden« nicht erteilt. Davon betroffen sind neben Gewerbetreibenden auch Rechtsanwälte, die nach Ausschluss aus der Anwaltschaft zunächst als nichtanwaltliche Rechtsberater hatten weiterarbeiten können. ${ }^{23}$ Voraussetzung für die Erteilung der Erlaubnis nach dem HPG ist gem. $\int 2$ Ic der Ersten Durchführungsverordnung (I. DVO HPG), ${ }^{24}$ dass Antragsteller und Ehegatte »deutschen oder artverwandten Blutes « sind, sowie die politische und sittliche Zuverlässigkeit des Antragstellers ( $\mathbb{2}$ If der I. DVO HPG). ${ }^{25}$

Das Ziel der Monopolisierung der Berufsausübung wird besonders deutlich im HPG. Eine Erlaubnis wird grundsätzlich nur an den erteilt, »der die Heilkunde bisher berufsmäßig ausgeübt hat «( 2 I HPG) oder sich »zur Zeit auf den Schu-

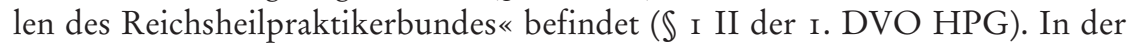
Gesetzesbegründung heißt es dazu: »In Zukunft [d.h. nach Ablauf der Antragsfrist am I. April I939] wird eine Erlaubnis zur Berufsausübung als Heilpraktiker nicht mehr erteilt werden. $\ll^{26} \mathbb{4}$ HPG verbietet es, »Ausbildungsstätten für Per-

I7 Gillhausen (Fn. I I), S. 2 I.

I 8 Seit I 874 dürfen Impfungen mit Schutzpocken nur durch »Impfärzte« durchgeführt werden (Reichsimpfgesetz v. 8.4.1874); seit I883 ist die Ausübung der Heilkunde »im Umherziehen « (sog. Wandergewerbe) verboten $(\mathbb{5} 6 \mathrm{a}$ GewO); seit 1900 ist die Behandlung seuchenbehafteter Patienten (Gesetz zur Bekämpfung gemeingefährlicher Krankheiten v. 30.6.1900), seit 1927 die Behandlung von Geschlechtskrankheiten Ärzten vorbehalten (Gesetz zur Bekämpfung von Geschlechtskrankheiten v. I 8.2.1927). Seit I 924 werden Leistungen von Nichtärzten nicht mehr von der gesetzlichen Krankenversicherung erstattet ( $\mathbb{S}$ I 22 Reichsversicherungsordnung i.d.F. v. I 5.I2.1924). Seit I938 ist die Geburtshilfe Ärzten und geprüften Hebammen vorbehalten (Hebammengesetz v. 2 I.I2.1938).

I9 Gesetz zur Verhütung von Missbräuchen auf dem Gebiet der Rechtsberatung v. I3.I 2.I935 (RGBl. I, S. 1478 ).

20 Gesetz über die berufsmäßige Ausübung der Heilkunde ohne Bestallung (Heilpraktikergesetz) v. I7.2.1939 (RGBl. I, S. $25 \mathrm{I})$.

2 I Für die Rechtsberatung: Raeke, JW I936, S. I; für die Heilbehandlung: Gesetzesbegründung zum Heilpraktikergesetz (Reichs- und Staatsanzeiger Nr. 50 vom 28.2.1939, S. 2).

22 Ausführungsverordnung vom I3.I2.1935 (RGBl. I, S. I48I).

23 Durch das Gesetz über die Zulassung zur Rechtsanwaltschaft vom 7.4.1933 (RGBl. I, S. I 88) wurden rassisch und politisch missliebige Personen aus der Rechtsanwaltschaft ausgeschlossen (bzw. ihnen der Zugang versperrt). Durch die Fünfte Verordnung zum Reichsbürgergesetz v. 27.9.1938 (RGBl. I, S. I I 46 ff.) wurde Juden endgültig der Beruf des Rechtsanwalts »verschlossen « (\$I). Gem. $\$ 8$ konnten sie als »jüdische Konsulenten« zur Beratung und Vertretung von Juden zugelassen werden.

24 Erste Durchführungsverordnung zum HPG v. I 8.2.1939 (RGBl. I, S. 259).

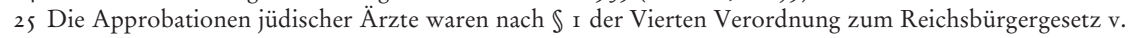
25.7.1938 (RGBl. I, S. 969) bereits am 30.9.1938 erloschen. Nach $\ 2$ konnte ihnen die Genehmigung erteilt werden, Juden zu behandeln. Demjenigen, der nicht in Besitz einer solchen Genehmigung war, war es nach $\rrbracket_{3}$ I verboten, die Heilkunde auszuüben.

26 Aus der Begründung zum Heilpraktikergesetz (Reichs- und Staatsanzeiger Nr. 50 vom 28.2.I939, S. 2). 
sonen, die sich der Ausübung der Heilkunde im Sinne dieses Gesetzes widmen wollen, einzurichten oder zu unterhalten. « 2 II HPG sieht stattdessen für jüngere, besonders geeignete Personen die Zulassung zum Medizinstudium »unter erleichterten Bedingungen « vor.

Während einige Heilpraktikerverbände im HPG noch immer die Geburtsstunde des Heilpraktikerberufs sehen, ${ }^{27}$ erweist es sich bei genauerer Betrachtung für Nichtärzte als Berufszugangssperre mit einer besitzstandswahrenden Übergangsregelung. Mit Erlöschen der letzten Erlaubnis wäre der Heilpraktikerberuf ausgestorben, an seine Stelle ein Ärztemonopol getreten. ${ }^{28}$

Etwas zurückhaltender ist das vier Jahre zuvor erlassene RBerG: Anders als im HPG wird weder eine generelle Zulassungssperre noch ein Ausbildungsverbot

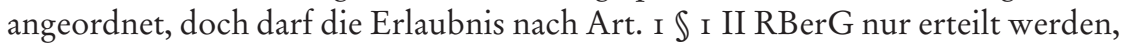
»wenn [...] das Bedürfnis nicht bereits durch eine hinreichende Zahl von Rechtsberatern gedeckt ist «. Jonas, Ministerialrat im Reichsjustizministerium und Verfasser des ersten Kommentars zum neuen RBerG, erläutert das Ziel dieser Regelung wie folgt:"29 Grundgedanke sei die »allmähliche Zurückführung « der Zahl der "gewöhnlichen Rechtsberater « auf den »Stand des künftigen wirklichen Bedürfnisses «. Dieses bestimme sich in erster Linie »nach den Interessen der die Rechtsbesorgung in Anspruch nehmenden Volksgenossen«. Dabei müssten die Rechtsanwälte und die von ihnen verwalteten NS-Rechtsbetreuungsstellen ${ }^{\circ}$ maßgeblich in Rechnung gestellt werden. ${ }^{3 \mathrm{I}}$ Es sei daher » wesentlich, daß es einen von jung an auf das Rechtskonsulentenwesen eingestellten Nachwuchs nicht gibt.« Der zukünftige Bedarf könne stattdessen durch »ältere und erfahrene Angestellte« im Bürodienst der Anwälte und Notare gedeckt werden.

Etwas anderes solle nur für »Spezialisten « gelten, deren (Teil-)Erlaubnis auf ein bestimmtes Sachgebiet zu beschränken sei. Gedacht ist - neben dem »ausländischen Volljuristen« - vor allem an die Grenzgebiete zum »Wirtschaftlichen«. Hier könne das Bedürfnis nach »Spezialsachverständigen wirtschaftsjuristischer Art « auch von den in Betracht kommenden Rechtsanwälten nicht oder nicht voll befriedigt werden. ${ }^{32}$ In den folgenden Jahren werden spezielle Regelungen für Inkassobüros, 33 Frachtprüfer 34 und Versicherungsberater ${ }^{35}$ erlassen.

Ein Bedarf nach nichtanwaltlichen Rechtsbeiständen wurde also nur dort anerkannt, wo Rechtsanwaltschaft und NS-Rechtsbetreuungsstellen die Nachfrage nach Rechtsrat nicht vollständig decken konnten. Die Entscheidung darüber, ob eine solche Nachfrage bestand, lag dabei in der Hand der erlaubniserteilenden Behörde.

27 Pressemitteilung des Fachverbandes Deutscher Heilpraktiker unter www.heilpraktiker.org/FDHPresse/pri 8.htm (Zugriff: 5.2.2007).

28 Der Entwurf eines Heilpraktikergesetzes aus dem Jahr 1933 hatte noch die Schaffung eines Standes der sog. approbierten Heilpraktiker vorgesehen: Gillhausen (Fn. I I), S. 26.

29 Jonas, Martin: Das Gesetz zur Verhütung von Missbräuchen auf dem Gebiete der Rechtsberatung, Berlin I936, S. I7 ff.

30 Noch in der Weimarer Republik bestand ein weitgefächertes Angebot von kirchlichen, gewerkschaftlichen und öffentlichen Rechtsauskunftsstellen für sozial Schwache, deren Ursprünge bis in die Kaiserzeit zurückreichten. I 934 wurde das »Amt für Rechtsbetreuung des Deutschen Volkes « gegründet, dem die in »NS-Rechtsbetreuungsstellen« umbenannten Rechtsauskunftsstellen unterstellt wurden. Ihre Tätigkeit war vom Anwendungsbereich des RBerG ausgenommen (Art. I $\ 3$ Nr.I in der Fassung von I935).

3 I Jonas (Fn. 29), S. 65 .

32 Jonas (Fn. 29), S. i 9 f.

33 Dritte Verordnung v. 25.6.1936 (RGBl. I, S. 514) sowie Allgemeinverfügung v. 24.I0.I94I (DJ I94I, S. 368).

34 Vierte Verordnung v. I 3.4.1937 (RGBl. I, S. 456).

35 Allgemeinverfügung v. 5.7.1938 (DJ 1938, S. I I I 4.) 
Beide Gesetze rechtfertigen die Aufhebung der Gewerbefreiheit mit dem

Schutzbedürfnis des Rechtsuchenden bzw. Kranken: Die Besorgung fremder Rechtsangelegenheiten sei weitgehend in die Hand von Personen geraten, die keine hinreichende Gewähr für Sachkunde und Zuverlässigkeit böten. Gleichwohl übe dieses »Winkelkonsulententum « auf die Rechtsuchenden eine bedenklich starke Anziehungskraft aus. $\mathrm{Zu}$ deren Schutz sei ein staatlicher Konzessionszwang erforderlich, da sich die Untersagungsmöglichkeit des $\ 35$ GewO als unzulänglich erwiesen habe. ${ }^{6}$ Auch die Kurierfreiheit habe zur Folge, dass sich »sachlich unfähige und charakterlich minderwertige Personen « auf diesem Gebiet betätigten und durch unzweckmäßige Behandlungsmethoden gesundheitliche Schäden anrichteten. Eine einwandfreie gesundheitliche Betreuung des deutschen Volkes sei daher sicherzustellen. 37 Einen Hinweis auf Ermittlungen, auf denen diese Annahmen beruhen könnten, sucht man in den knappen Gesetzesbegründungen vergebens. ${ }^{38}$

Als schutzbedürftig gilt neben der reibungslosen Arbeit der Behörden nun auch die Anwaltschaft. Diese müsse vor Wettbewerb mit Personen, die gleichartigen berufs- und standesrechtlichen Beschränkungen (wie z.B. der Bindung an eine Gebührenordnung) nicht unterworfen sind, bewahrt werden. Dabei wird ausdrücklich auf die wirtschaftliche Lage der Anwaltschaft Bezug genommen und das »sachliche Bedürfnis « nach Rechtsbeiständen von ihr abhängig gemacht. 39

Diese Lage - genauer: die » wirtschaftliche Notlage der Anwaltschaft « - war auch zentraler Gegenstand einer Tagung anwaltlicher Berufsvertreter des Bundes Nationalsozialistischer Juristen am 22. November 1935. Anstelle der Zwangspensionierung aller über 65jährigen Anwälte, der Einführung einer Rechtsschutz-Sozialversicherung oder der Erhöhung der Armenrechtsgebühr wurde den Rechtsanwälten von Seiten des Reichsjustizministeriums - neben der Einschränkung des freien Zugangs zur Anwaltschaft und einer beschleunigten Durchführung der Nürnberger Gesetze auch im Bereich der Anwaltschaft - die baldigste Verabschiedung eines »Schwarzarbeitergesetzes" (gemeint ist das Rechtsberatungsgesetz) in Aussicht gestellt. $4^{\circ}$

Nach ihrer »Entnazifizierung «4I durch das Kontrollrats-Gesetz Nr. ${ }^{42}$ und die Kontrollrats-Proklamation Nr. $3^{43}$ galten beide Gesetze in der Bundesrepublik fort. Während Bedürfnisprüfung (im RBerG) ${ }^{44}$ und Zulassungssperre (im

36 Aus der Begründung zum Rechtsberatungsgesetz (Reichssteuerblatt 1935, S. I 528 ).

37 Aus der Begründung zum Heilpraktikergesetz (Reichs- und Staatsanzeiger Nr. so vom 28.2.1939, S. 2).

38 Für Jonas (Fn. 29), S. 4, stand die Unzulänglichkeit der bisherigen Regelung »bei den Sachkundigen« seit langem außer Streit. Konkrete Hinweise auf Beschwerden über oder Schäden durch Rechtsberater findet man auch bei ihm nicht.

39 Aus der Begründung zum Rechtsberatungsgesetz (Reichssteuerblatt I935, S. I 528 ): »Nur so ist es möglich [...] die Zahl der dem Berufe angehörenden in den den sachlichen Bedürfnissen entsprechenden Grenzen zu halten. In letzterer Hinsicht darf vor allem auch die wirtschaftliche Lage der Anwaltschaft nicht außer acht gelassen werden «.

40 Die Tagung ist dokumentiert in: JW I935, S. 3448 ff.

4I Dazu eingehend: Rottleuthner, Zur Entstehungsgeschichte und den Zielen des Gesetzgebers 1935: http:/ /www.rechtsberatungsgesetz.info/gesetzgebung/entstehung.html (Zugriff: 5.2.2007).

42 Art. I Nr. I des Gesetzes v. I0.I0.I945 erklärte die NSDAP und ihre Gliederungen für abgeschafft und ungesetzlich (KRAB1. S. I9). Seitdem läuft die Erlaubnisfreiheit von Rechtsberatung und Rechtsbetreuung durch »Dienststellen der NSDAP und ihrer Gliederungen « (und somit auch der NS-Rechtsbetreuungsstellen) ins Leere, wovon noch heute die »... « in $\ 3$ Nr. I RBerG einiger Gesetzessammlungen (z.B. des »Schönfelder«) erinnern.

43 Art. I der Proklamation v. 20.10.1945 verbietet die Ungleichbehandlung aufgrund von Rasse, Staatsangehörigkeit oder Religion (KRABl. S. 22). Eine explizite Änderung von RBerG und HPG erfolgte nicht; das war Sache der Gerichte.

44 Urteil des BVerwG v. 5.5.1955 (NJW 1955, I 532). Eine Bedürfnisprüfung ist heute nur noch bei Personen zulässig, die keine »Deutschen« i.S.d. Art. I I6 GG und keine Angehörigen eines EG-Staates oder EWR-Staates sind. 
HPG ${ }^{45}$ vom Bundesverwaltungsgericht (BVerwG) für mit Art. I 2 GG unvereinbar erklärt wurden, blieb das Verbot mit Erlaubnisvorbehalt in beiden Fällen erhalten. Damit waren jedoch entgegen dem ursprünglichen Gesetzeszweck aus RBerG und HPG »normale« Berufsordnungsgesetze geworden. Nach der Entscheidung des Bundesverwaltungsgerichts war der Weg frei für eine allein von den Eigenschaften und Fähigkeiten des Antragstellers abhängige Erlaubniserteilung.

Doch welche Voraussetzungen muss der Antragsteller für eine Erlaubniserteilung nachweisen? Es hat sich gezeigt, dass die Erlaubnis in HPG und RBerG lediglich als Übergangslösung bzw. »Lückenfüller« geplant war. Zwar erfolgte die Überprüfung durch eine staatliche Stelle; ${ }^{6}$ ihr Ziel war jedoch lediglich die Abwehr von Gefahren für Rechtsuchende bzw. Kranke. Eine Regelung der Ausbildung schien daher 1935/1939 ebenso wenig angebracht wie eine nähere Ausgestaltung der Prüfung. Das HPG stellte zunächst lediglich auf die »berufliche Eignung « ( 2 I g der I. DVO HPG) ab, ohne diese näher zu konkretisieren. Ab I94 I wurde die Erlaubnis dann nicht mehr erteilt, »wenn sich aus einer Überprüfung der Kenntnisse und Fähigkeiten des Antragstellers ergibt, dass die Ausübung der Heilkunde durch den Betreffenden eine Gefahr für die Volksgesundheit bedeuten würde. «( $\int 2$ Ii der 2. DVO HPG).47 Nach $\ 8$ der I. AVO RBerG hatte der Nachsuchende seine Sachkunde und Eignung durch Angaben über seinen Ausbildungsgang und seine bisherige berufliche Tätigkeit darzulegen. Negativ sei damit zum Ausdruck gebracht, so Jonas, ${ }^{48}$ dass die Behörde keine Anwärterprüfung abhalten solle. Ziel der Überprüfung war in beiden Fällen lediglich die Gefahrenabwehr. Ein staatlicher Befähigungsnachweis sollte aus der Erlaubnis dagegen gerade nicht hergeleitet werden können.49 An dieser Situation hat sich trotz der grundlegenden Wandlung beider Gesetze nach 1945 wenig geändert. Bis heute sind die fachlichen Voraussetzungen für die Erlaubniserteilung im HPG und RBerG nur rudimentär geregelt. Welche Kenntnisse erforderlich sind, um zu verhindern, »daß die Ausübung der Heilkunde durch den Betreffenden eine Gefahr für die Volksgesundheit bedeuten würde , $5 \circ$ bleibt ebenso offen wie die Frage, was die für die jeweilige Teilerlaubnis erforderliche »persönliche Eignung sowie genügende Sachkunde« voraussetzt. ${ }^{I}$ Dass sich auch die Bundesländer, denen die Ausführung der Gesetze obliegt, nicht auf eine einheitliche Konkretisierung der bundesrechtlichen Vorgaben verständigt haben, zog eine höchst unterschiedliche Überprüfungspraxis nach sich. ${ }^{52}$

45 Urteil des BVerwG v. 24.I.I957 (NJW I957, 84I).

46 RBerG: der Präsident des zuständigen Amts- bzw. Landgerichts ( $\$$ I I der I. AVO zum RBerG); HPG:

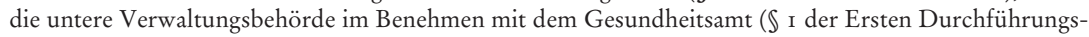
verordnung zum HPG v. I 8.2.1939, RGBl. I, S. 259).

47 Zweite Durchführungsverordnung v. 3.7.I94I (RGBl. I, S. 368).

48 Jonas (Fn. 29), S. 64.

49 Jonas (Fn. 29), S. I6 hält es daher für unzulässig, wenn der Rechtsberater die Erlaubnis zu Werbezwekken hervorhebt.

50 $\ 2$ der Ersten Durchführungsverordnung zum HPG v. I 8.2.1939 (RGBl. I, S. 259).

5I Art. I $\$$ I II I RBerG. Aus $\$ 8$ der Ausführungsverordnung zum RBerG v. I3.I 2.1935 (RGBl. I, S. I48 I), wonach der Nachsuchende seine Sachkunde und Eignung durch Angaben über Ausbildung und Berufstätigkeit darzulegen hat, wird geschlossen, dass der Antragsteller neben theoretischem Wissen auch über praktische Erfahrungen verfügen muss. (Rennen, Günter/Caliebe, Gabriele: RBerG, 3. Aufl., München 200 I, $\int 8$ I. AVO Rn. 4).

52 Arndt, Klaus Friedrich: Heilpraktikerrecht: Entstehung, Funktionswandel, Berufszulassungsregelung, Frankfurt am Main 1985, S. 73 ff.; Franz, Kurt: Naturheilmittel und Recht, Köln 1993, S. I65, wonach es »auch schon einmal ausreichend sein kann, wenn der Bewerber lediglich Kenntnisse von den einschlägigen Rechtsvorschriften hat.« Auf die uneinheitliche Überprüfungs- und Aufsichtspraxis weist auch die Gesetzesbegründung zum RDGE hin: BR-Drs. 623/06, S. 87. 
In den Schwierigkeiten, die fachlichen Anforderungen an Heilpraktiker und Rechtsberater zu bestimmen, zeigt sich wiederum der Einfluss der Profession. Sie setzt den Maßstab, an dem sich auch Nichtprofessionelle mit gleichem Tätigkeitsspektrum zu orientieren haben. So weist die Bundesregierung I 983 in ihrer Antwort auf die Frage, welche Anforderungen an die Ausbildung der Heilpraktiker zu stellen seien, auf die Probleme hin, denen sie sich bei einer gesetzlichen Regelung von Ausbildung und Prüfung gegenübergestellt sähe. Aufgrund der umfassenden Berechtigung der Heilpraktiker zur Ausübung der Heilkunde müsste eine solche Regelung den für die Berufsbildung der Ärzte bestehenden Vorschriften nahe kommen und würde damit zugleich die Frage nach der Existenzberechtigung zweier vergleichbarer Heilberufe aufwerfen. 53

So entstanden durch die Verkehrung des ursprünglichen Gesetzeszweckes mit Heilpraktiker und Rechtsbeistand zwei »Scheinprofessionen «, die zwar hinsichtlich ihrer Lizenzierung, also der Berechtigung, Tätigkeiten auszuführen, die anderen verboten sind, den Professionen Arzt und Rechtsanwalt entsprechen, jedoch weder an vergleichbare Berufszugangs- 54 noch Berufsausübungsregelnss gebunden sind.

Gilt dies für das HPG bis heute, schlug I 980 die Stunde der Rechtsbeistände. ${ }^{6}$ Die »Schließung des Berufs der Vollrechtsbeistände« kam erstmals gegen Ende der Legislaturperiode im Juni I980 im Rechtsausschuss des Bundestages während der Beratungen eines anderen Gesetzgebungsvorhabens zur Sprache. Die Abgeordneten Kleinert (FDP) und Schöfberger (SPD) begründeten ihren Antrag mit dem Wegfall eines Bedürfnisses nach Vollrechtsbeiständen, d.h. nach Inhabern einer uneingeschränkten Erlaubnis nach dem RBerG. Angesichts der steigenden Komplexität des Rechts sei es vielmehr sinnvoll, nur noch Teilerlaubnisse für fünf abschließend aufgezählte Sachbereiche (Rentenberater, Frachtprüfer, vereidigte Versteigerer, Inkassounternehmer und Rechtskundige in einem ausländischen Recht) 57 zu erteilen. Auch hätten die Standesorganisationen der Rechtsanwälte sowie der Rechtsbeistände ${ }^{8}$ auf eine solche Regelung gedrungen. Dem Vorwurf, der Rechtsausschuss dürfe sich nicht zum Lobby-Ausschuss für die Anwaltschaft machen, wurde als weiterer »Hauptgrund « für die Änderung der Missstand sog. »Rechtsbeistandsschulen« entgegengehalten. Hier würden Nichtjuristen in einem halben Jahr auf die Zulassung als Rechtsbeistand vorbereitet. Für Rechtsuchende bestehe die Gefahr der Verwechselung mit demjeni-

53 Antwort der Parl. Staatssekretärin Frau Karwatzki auf Frage des MdB Faltlhauser (BT-Drs. Io/6r6, Frage 4I, 42) in der 37. Sitzung des Deutschen Bundestages am 24. I I.I 983 , Sten. Ber. S. 2622 (A).

$54 \int \mathbb{S} 4 \mathrm{ff}$. Bundesrechtsanwaltsordnung i.V.m. $\int \mathbb{S} 5 \mathrm{ff}$. Deutsches Richtergesetz sowie den landesrechtlichen Vorschriften über die juristische Prüfung und Ausbildung; $\mathbb{S} 3$ ff. der Bundesärzteordnung i.V.m. der Approbationsordnung für Ärzte.

55 Während Gesetzgeber und berufsständische Körperschaften die Berufsausübung von Arzt und Rechtsanwalt durch eine Vielzahl verbindlicher Vorschriften geregelt haben (nur beispielhaft seien Zeugnisverweigerungsrecht, Verschwiegenheitspflicht und Gebührenordnungen genannt), fehlen vergleichbare Bindungen bei nichtprofessionell Tätigen. Berufsrechtliche Vorschriften existieren hier nur auf der Ebene des Privatrechts (so z.B. als Satzungen der Berufsverbände).

56 Art. 2 VI des Fünften Gesetzes zur Änderung der Bundesgebührenordnung für Rechtsanwälte vom I 8.8.1980 (BGBl. I, S. I 503 , I 507 ).

57 Der bereits etablierte Beruf der »Versicherungsberater « wurde zunächst vergessen und musste 1989 aufgrund des Beschlusses des BVerfG v. 5.5.1987, BGBl. I, S. 250 I = NJW I988, 543, nachträglich eingefügt werden: Art. 3 des Gesetzes zur Änderung des Berufsrechts der Rechtsanwälte und Patentanwälte vom I 3. Dezember 1989 (BGBl. I, S. 2 I 47).

58 Es scheint nicht ausgeschlossen, dass dieses »Drängen« von Seiten der Rechtsbeistände davon beeinflusst war, dass aufgrund einer gleichzeitigen Änderung von $\ 209$ BRAO (Voll)Rechtsbeistände »auf Antrag in die für den Ort ihrer Niederlassung zuständige Rechtsanwaltskammer« aufgenommen wurden. 
gen, der zwei Staatsexamina abgelegt habe.59 Keine dieser Behauptungen wurde im Verlauf des Gesetzgebungsverfahrens einer empirischen Überprüfung unterzogen.

Damit kehrt sich die Entwicklung für das RBerG I980 wieder um. Der bundesdeutsche Gesetzgeber erklärt - in Fortführung des Zieles des historischen Gesetzgebers - das Bedürfnis nach Vollrechtsbeiständen für erloschen und errichtet angesichts der dem Verbraucher anderenfalls drohenden Gefahren für die umfassende Rechtsberatung ein Anwaltsmonopol.

\section{Nichtprofessionelle Tätigkeit: Gefabren und Bedürfnisse}

\section{Gefabren}

Mit Verweis auf die professionellen Anforderungen an Berufszugang und Berufsausübung lassen sich abstrakte Gefahren für den Verbraucher leicht plausibel machen. Dagegen fällt der Nachweis konkreter Schäden durch nichtprofessionell Tätige ungleich schwerer. Empirische Studien zu dieser Frage fehlen. Die Bundesregierung antwortete I984 auf die Frage nach den in den vergangenen zehn Jahren mit dem HPG gemachten Erfahrungen: »Über Schadensfälle, die durch Heilpraktiker verursacht werden, ist hier, obwohl das Bundesministerium für Jugend, Familie und Gesundheit dieser Frage intensiv nachgegangen ist, wenig bekannt. ${ }^{60}$

In der Begründung des RDGE wird die Aufrechterhaltung eines Verbots mit Erlaubnisvorbehalt dagegen mit den »oft weit reichenden Folgen unqualifizierten Rechtsrats « begründet. ${ }^{6}{ }^{1}$ Nähere Angaben zu Art und Umfang dieser »Folgen « sucht man in dem 230 Seiten umfassenden Entwurf jedoch vergeblich. Zwar berichten Rechtsanwaltskammern und Anwaltsvereine von zahlreichen Verfahren gegen »illegale« Rechtsberater; ob diese Personen aber, abgesehen von einem Verstoß gegen die Erlaubnispflicht des RBerG, auch Schäden für den Verbraucher zu verantworten haben, wird meist nicht bekannt.

Als Grund für das Fehlen entsprechender Dokumentationen kommt zum einen die Besonderheit professioneller Tätigkeit selbst in Betracht. So verweist die Gesetzesbegründung darauf, dass im Rechtsdienstleistungsbereich »in weiterem Umfang als in anderen Lebensbereichen eine Asymmetrie der Informationen zwischen Auftraggeber und Auftragnehmer « bestehe. ${ }^{62}$ Mit anderen Worten: Der Verbraucher - unabhängig davon, ob Asylbewerber oder Unternehmer sei nicht oder nur bedingt in der Lage, die Qualität der Dienstleistung zu beurteilen. Er könne die Rechtsberatung nicht »in die Hand nehmen « und mit anderen Angeboten vergleichen. ${ }^{63}$ Fehler und daraus resultierende Schäden könnten ihm daher verborgen bleiben. Dabei stellt sich jedoch die Frage nach dem Maßstab, der an eine nichtprofessionelle Dienstleitung zu stellen ist. Ist die Leistung bereits mangelhaft, wenn sie hinter den professionellen Standards zurückbleibt? Oder sind bei der Beurteilung der Qualität auch die individuellen Umstände des

59 Gesetzesmaterialien zum Fünften Gesetz zur Änderung der Bundesgebührenordnung für Rechtsanwälte, 8. Wahlperiode I976, lfd. Nr. I6 (Stenographisches Protokoll über die 99. Sitzung des Rechtsausschusses am i 8.6.1980, S. is f.).

60 Antwort der Parl. Staatssekretärin Frau Karwatzki auf Frage des MdB Faltlhauser (BT-Drs. I0/I 539, Frage 48, S. 23).

6I BR-Drs. 623/06, S. 58 .

62 BR-Drs. $623 / 06$, S. 58.

63 Finzel, Dieter: Referat auf dem 65. Deutschen Juristentag 2004, Abteilung Rechtsberatung, in: Verhandlungen des 65. Deutschen Juristentages Bonn 2004, Band II/I, München 2004, S. R27 ff. 
Einzelfalls wie z.B. die vereinbarte Vergütung oder eine für den Verbraucher

erkennbar geringere Ausbildung des Beraters zu berücksichtigen?

Damit ist eine andere mögliche Ursache angesprochen: Danach könnten die fehlenden Schadensfälle darauf zurückzuführen sein, dass der Verbraucher in stärkerem Maße als vom Gesetzgeber vermutet in der Lage ist, zwischen unterschiedlich qualifizierten Anbietern zu unterscheiden. Dass er die Qualität der Dienstleistung womöglich nicht vollständig beurteilen kann, wohl aber zwischen Rechtsanwalt und Rechtsberater, Arzt und Heilpraktiker zu unterscheiden weiß. ${ }^{64}$

Darüber hinaus sollen mit dem RDGE nicht nur Behörden und Gerichte, sondern auch Rechtsordnung und Rechtskultur geschützt werden. Dem liegt die nicht weiter begründete Vorstellung zugrunde, »Recht « dürfe als höchstrangiges Gemeinschaftsgut »grundsätzlich nicht in die Hände unqualifizierter Personen gelangen «.65 Dies steht jedoch im Widerspruch zur Beschränkung des Anwendungsbereichs des RDGE auf außergerichtliche Rechtsdienstleistungen: Überall dort, wo es nicht um eine verbindliche Entscheidung durch die Rechtspflege geht, ist Recht ein Kulturgut, das bei seiner Anwendung durch den Bürger nicht etwa Schaden nimmt, sondern in der Gesellschaft »lebt « und sich mit ihr entwikkelt.

\section{Bedürfnisse}

In ihrer Koalitionsvereinbarung vom I6. Oktober 2002 haben SPD und Bündnis 90/Die Grünen beschlossen, das RBerG »den gesellschaftlichen Bedürfnissen « anzupassen. Dabei erweist es sich methodisch als schwierig, Bedürfnisse nach einer Tätigkeit zu ermitteln, die nach geltendem Recht weitgehend verboten ist. Das »Forschungsprojekt Rechtsberatungsgesetz « hat sich daher nicht mit der »Nachfrage-«, sondern mit der »Angebots-Seite« des Rechtsberatungsmarktes befasst und die Bedürfnisse und Probleme der Anbieter ermittelt. Diese wurden nicht nur insoweit analysiert, als sie in der Tätigkeit des Gesetzgebers, der Behörden und Gerichte ihren Niederschlag gefunden haben, sondern es wurde darüber hinaus die Befragung von über so Stellen, Organisationen, Berufs- und Interessenverbänden ausgewertet.

\section{a) Bedürfnis nach nichtprofessionellen Dienstleistungen}

Hier bietet sich aufgrund der abweichenden Entwicklung des HGP der Heilpraktiker als Vergleichsobjekt an. So hat das Bundesverfassungsgericht I 988 ausgeführt, das HPG erfasse ein »Berufsfeld «, ohne nach einzelnen Berufsbildern zu differenzieren. ${ }^{66}$ Bleibt man in diesem Bild, so lässt sich feststellen, dass auf dem »Berufsfeld « des HPG alternative, wissenschaftlich (noch) nicht fundierte und gesetzlich geregelte Heilmethoden wie etwa die Homöopathie, die Chirotherapie, ${ }^{67}$ Psychotherapie ${ }^{68}$ oder die Akupunktur »gedeihen« konnten. Nach

64 Dagegen geht die Bundesregierung (wie bereits der Gesetzgeber I980) in der Begründung des RDGE davon aus, dass den Rechtsuchenden »das Nebeneinander zweier auf die gleiche Tätigkeit ausgerichteter Rechtsberatungsberufe mit völlig unterschiedlicher Berufsqualifikation« nicht zu vermitteln wäre (BRDrs. 623/06, S. 60).

65 BR-Drs. 623/06, S. 92.

66 BVerfG io.5.1988, NJW i $988,2290$.

67 BVerwG 25.6.1970, NJW 1970, 1987: Tätigkeit als Chiropraktiker (erlaubnispflichtige) Ausübung der Heilkunde i.S.d. HPG.

68 Urteil des BVerwG 10.2.1983, NJW 1984, I4I4: Psychotherapie (erlaubnispflichtige) Ausübung der Heilkunde i.S.d. HPG. Für Diplom-Psychologen, die selbständig therapeutisch tätig sein wollten, war die Erlaubnis nach dem HPG bis 1998 die einzige Alternative zu einem Medizinstudium. 
wissenschaftlicher Bestätigung bildeten sich daraus z.T. neue Berufe, ${ }^{69}$ z.T. wurden Heilmethoden (wie z.B. Akupunktur oder Ozontherapie) von Teilen der ärztlichen Profession übernommen.

Im Fall des RBerG überließ der Gesetzgeber das »Berufsfeld « dagegen I 980 fast ausschließlich den Rechtsanwälten und verwandelte es weitgehend in eine »Monokultur«. Nur einzelne Spezialberufe wurden am Rand »unter Schutz« gestellt. Den Kampf gegen unerlaubte »Eindringlinge« übernehmen die Rechtsanwälte und sorgen durch Abmahnungen nach dem UWG und/oder Anzeigen bei der Staatsanwaltschaft für die Durchsetzung des Verbotes.

Durch die weite Fassung des Verbotstatbestandes konnten sich neue Berufe nur eingeschränkt oder von Rechtsanwälten dominiert entwickeln. Der Rechtsanwalt als Monopolist für die Beratung und Vertretung in allen Rechtsangelegenheiten (vgl. \3 I BRAO) wurde damit nicht nur weitgehend vor gewerbsmäßiger Konkurrenz geschützt. Jede Verrechtlichung von Lebensbereichen führt zudem zu einem »Landgewinn « für Anwälte, den diese gegen nichtanwaltliche Konkurrenz zu verteidigen suchen. So wurde es Mediatoren auf Antrag anwaltlicher Kläger hin untersagt, »an der außergerichtlichen Streitbeilegung mitzuwirken, ohne dass daran Personen, die Rechtsberatungen durchführen dürfen, beteiligt sind $« .70$ Das OLG Brandenburg verbot es einem Energieberater, Energieversorgungsunternehmen im Namen von Kunden aufzufordern, ihm Stromlieferverträge zur Prüfung vorzulegen. ${ }^{7 \mathrm{I}}$ In anderen Fällen wie z.B. bei Patentüberwachungsunternehmen ${ }^{72}$ oder Erbenermittlern 73 stellte schließlich (erst) das Bundesverfassungsgericht klar, dass Tätigkeiten, die auch in einer Rechtsanwaltskanzlei regelmäßig durch Hilfspersonen erledigt werden bzw. überwiegend auf wirtschaftlichem Gebiet liegen, nicht vom RBerG erfasst werden. Entsprechendes gilt für zahlreiche weitere Berufe, die an das Berufsfeld der Anwälte grenzen und mit diesen z.T. seit Jahrzehnten »Nachbarschaftsstreitigkeiten« führen. 74

Diesen von Karlsruhe vorgenommenen Einschränkungen des Anwendungsbereichs des RBerG wird vom RDGE zum einen mit dem neuen Begriff der »Rechtsdienstleistung « Rechnung getragen, der nur solche Tätigkeiten erfassen soll, die »eine besondere rechtliche Prüfung des Einzelfalls« erfordern. Nicht vom Anwendungsbereich erfasst wären danach Dienstleistungen, die keine Prüfung der Rechtslage im Sinn eines »spezifisch juristischen Subsumtionsvorgangs « voraussetzen.75 Zum anderen soll insbesondere wirtschaftlich tätigen Unternehmen in größerem Umfang als bisher Rechtsdienstleistung, die im Zusammenhang mit ihrer beruflichen Tätigkeit steht, als sog. »Nebenleistung « erlaubt werden. Darüber hinaus will die Bundesregierung dem Bedürfnis nach neuen Dienstleistungen, die Rechtsanwälte (bisher) nur selten allein anbieten, vor allem durch die Ermöglichung neuer Formen der »interprofessionellen Zusammenarbeit« entsprechen. So soll es Rechtsanwälten durch die Änderung

69 Gesetz über die Berufe des Psychologischen Psychotherapeuten und des Kinder- und Jugendlichentherapeuten v. I6.6.1998 (BGBl. I, S. I3 I I).

70 LG Rostock Ir.8.2000, NJW-RR 200I, I 290, bestätigt durch: OLG Rostock 20.6.2001, NJW-RR 2002, 642.

7I OLG Brandenburg I9.9.200I (7 U I09/OI). Liberaler: OLG Düsseldorf I 5.7.2003, NJW 2004, 489.

72 BVerfG 29.10.1997, NJW I998, 348I.

73 BVerfG 27.9.2002, NJW 2002, 353 I.

74 Ohne Anspruch auf Vollständigkeit seien Banken, Inkassounternehmen, Makler, Steuerberater und Unternehmensberater genannt. Eine Übersicht findet sich bei: Rottleuthner/Klose (Fn. 7), $\mathrm{H}_{24} \mathrm{ff}$.

75 BR-Drs. 623/06, S. 94. 
von $\int 59 \mathrm{a}$ BRAO ermöglicht werden, in einer gemeinsamen Sozietät mit einem nichtanwaltlichen Mediator oder Unternehmensberater zusammenzuarbeiten. ${ }^{76}$ Daneben sind jedoch die Bedürfnisse von Verbrauchern zu berücksichtigen, die das Angebot professioneller Dienstleister aus vielfältigen Gründen nicht wahrnehmen können oder wollen. Wie ein Blick auf das HPG zeigt, können dafür nicht allein finanzielle, sondern auch sprachliche und psychologische Hürden ausschlaggebend sein. Trotz einer flächendeckenden ärztlichen Versorgung in der Bundesrepublik, deren Kosten (noch) weitestgehend von den Krankenkassen übernommen werden, gibt es offenbar im medizinischen Bereich das Bedürfnis nach Alternativen. Schätzungen gehen davon aus, dass drei bis fünf Millionen Bundesbürger relativ regelmäßig zu einem Heilpraktiker gehen, obwohl dessen Kosten von der gesetzlichen Krankenkasse nicht erstattet werden.77 Als Gründe werden dabei vor allem die Enttäuschung von der Schulmedizin nach oft jahrelangen »Patientenkarrieren«, Kommunikationsschwierigkeiten (»Fachjargon«) und soziale Distanz zwischen Patient und Arzt (»Halbgott in Weiß «) genannt. ${ }^{8}$ Gerade der Umstand, dass es sich bei einem Heilpraktiker nicht um ein Mitglied der Profession handelt, scheint also Motiv für einen Besuch bei ihm zu sein.

Fragt man nach Hindernissen, die den Gang zum Anwalt erschweren, ist neben den hohen Kosten ( $58 \%$ ) und der Schwierigkeit, einen kompetenten Anwalt auszuwählen ( $52 \%$ ), für immerhin 29 \% der Befragten die Sorge, die Fachsprache der Anwälte nicht zu verstehen, ausschlaggebend. $25 \%$ befürchten, dass der Anwalt an kleineren Fällen nicht interessiert sei, und I $8 \%$ scheuen sich, einen Anwalt einzuschalten, weil dieser den Konflikt verschärfen und vor Gericht drängen werde.79

Soweit in diesen Antworten ein Bedürfnis nach einem subprofessionellen Dienstleistungsangebot zum Ausdruck kommt, findet dies im RDGE keine Berücksichtigung. Eine von der Anwaltschaft nicht gedeckte Nachfrage wird allein im Bereich der bereits bestehenden Teilerlaubnisse anerkannt, die als »registrierte Personen « Eingang in den RDGE gefunden haben. Die insbesondere von Seiten der Diplom-Wirtschaftsjuristen und Rechtsschutzversicherer geforderte Zulassung zur selbständigen Rechtsberatung wurde aus Gründen des Verbraucherschutzes abgelehnt. ${ }^{80}$ Einen mit dem Heilpraktiker vergleichbaren allgemeinen Rechtsdienstleistungsberuf unterhalb der Rechtsanwaltschaft soll es auch in Zukunft nicht geben.

\section{b) Bedürfnis nach unentgeltlichen Dienstleistungen}

Angesichts des Fehlens einer »Rechtsschutz-Sozialversicherung und des Desinteresses vieler Rechtsanwälte an der Vertretung Mittelloser ${ }^{8 \mathrm{I}}$ ist schließlich

76 BR-Drs. 623/06, S. I 82.

77 Donhauser, Hubert: Der Beruf des Heilpraktikers - Analyse der Ausbildungssituation und Vorschläge zur Verbesserung der Ausbildung, I996, S. 22.

78 Rogalla, Dorothea: Warum gehen Patienten zum Heilpraktiker, Hannover 1980. Danach gaben $83 \%$ der befragten Patienten an, vor dem Besuch des Heilpraktikers in ärztlicher Behandlung gewesen zu sein. Für $40 \%$ der Befragten war die mangelnde Kommunikation mit ihrem Arzt Grund für den Gang zum Heilpraktiker.

79 Ergebnisse einer nicht veröffentlichten repräsentativen Bevölkerungsbefragung von forsa / Prognos, 27. Februar bis 4. März 2004, n= I.003, S. I I. Zur ausgeprägten Scheu vor Gerichten und Rechtsanwälten gerade der unteren Schichten: Raiser, Thomas: Das lebende Recht, Baden-Baden 1999, S. 352 ff.

80 BR-Drs. 623/06, S. 59 ff. (Diplom-Wirtschaftsjuristen), S. Ios f. (Rechtsschutzversicherer).

8I Als Ursachen verweist der Deutsche Caritasverband auf die geringen Gebührensätze für Sozialrechtsfälle sowie auf die Scheu vor einer Konfrontation mit Drogensüchtigen, Wohnungslosen und psychisch Kranken in der eigenen Kanzlei. (Unveröffentlichte Stellungnahme des Deutschen Caritasverbandes gegenüber dem BMJ v. 23.7.2003, S. 2) 
nach Alternativen zu kommerziellen Rechtsberatungsangeboten zu fragen. ${ }^{82}$ Der Verbotstatbestand des Art. I $\int$ I I I RBerG erfasst jede »geschäftsmäßige« Besorgung fremder Rechtsangelegenheiten »ohne Unterschied zwischen entgeltlicher oder unentgeltlicher Tätigkeit«. Ausnahmen im RBerG sind entweder auf spezielle Rechtsgebiete oder (zusätzlich) auf den Kreis der Mitglieder beschränkt. So ist Behörden und Körperschaften (Art. I $\int 3$ Nr. I RBerG), Verbraucherzentralen (Art. I $\ 3$ Nr. 8 RBerG) und Schuldnerberatungsstellen (Art. I $\int 3$ Nr. 9 RBerG) die Beratung nur »im Rahmen ihrer Zuständigkeit « bzw. »ihres Aufgabenbereichs « gestattet. Die Befugnis sog. »berufsständischer Vereinigungen « (Art. I $\$ 7$ RBerG), worunter u.a. Mietervereine und Gewerkschaften fallen, ist auf Aufgabenbereich und Mitglieder beschränkt.

Für viele Ratsuchende (z.B. Alkohol- und Drogenabhängige, Obdachlose und Migranten) bleibt daher nur die Rechtsberatung als Teil der Sozialberatung, die in erster Linie von den Trägern der Freien Wohlfahrtspflege angeboten wird. Sozialarbeiter dürfen zwar über Ansprüche aus dem Bundessozialhilfegesetz und - soweit für eine sachgerechte Hilfe erforderlich - auch aus anderen Sozialgesetzen aufklären und im behördlichen Verfahren Unterstützung leisten. Lehnt die Behörde den Anspruch jedoch ab, ist die Vertretung durch den Sozialarbeiter im behördlichen Widerspruchsverfahren als Verstoß gegen das RBerG verboten. ${ }^{8}$ Auch die Vorformulierung eines Duldungsantrags ist ihm als Behandlung einer Rechtsfrage »in sonstigen Rechtsgebieten « verboten. Während sich die Mitarbeiter der Freien Wohlfahrtspflege angesichts der ungeklärten Rechtslage häufig in einem Graubereich bewegen, ${ }^{84}$ stehen private Hilfsorganisationen und Initiativen (von amnesty international bis zur Deutschen Alzheimergesellschaft) mangels einer entsprechenden Ausnahme im RBerG mit beiden Beinen in der Illegalität. ${ }^{85}$

Dies gilt erst recht für Einzelpersonen: Während bereits in der Begründung des HPG I939 ausdrücklich darauf hingewiesen wurde, dass, »wer in selbstloser Weise seinen Mitmenschen hilft, Krankheiten vorzubeugen oder sie zu heilen«, daran weder gehindert werden könne noch solle, kam das OLG Oldenburg I99I zu dem Schluss, dass ein Sohn, der für seine Mutter in drei Fällen vor dem Amtsgericht als Prozessbevollmächtigter aufgetreten war, dem RBerG zuwider gehandelt habe. ${ }^{86}$ Auch angesichts der juristischen Kenntnisse und der langjährigen Berufserfahrung eines pensionierten Richters wurden die Gefahren für den Verbraucher gegenüber dem Bedürfnis nach altruistischer Rechtsberatung als höherwertig eingeschätzt. Seine Verurteilung aufgrund einer Selbstanzeige wegen unerlaubter Rechtsberatung wurde erst durch einen Beschluss des Bundesverfassungsgerichtes aufgehoben. ${ }^{87}$ Bereits das Schreiben an den Vermieter der Lebensgefährtin, der Brief an die gegnerische Haftpflichtversicherung eines Bekannten oder das Auftreten bei Behörden oder vor Gericht kann eine »Anzeige« bei der örtlichen Anwaltskammer zur Folge haben. Es folgen

82 Zur Rechtsberatung für sozial Schwache: Rasehorn, Theo (Hrsg.): Rechtsberatung als Lebenshilfe, Neuwied/Darmstadt 1979.

83 OVG Münster 29.II.200I, NJW 2002, I442.

84 Eingehend: Rottleuthner/Klose (Fn. 7), $\mathrm{H}_{5}$ I ff.

85 Beispielhaft erwähnt sei hier die Verurteilung des Geschäftsführers eines Vereins, der sich für ausländische Flüchtlinge engagiert hatte, im Jahr $200 \mathrm{I}$ zu einer Geldbuße i.H.v. 900,- DM. Das Verfahren ist dokumentiert unter: www.rechtsberatungsgesetz.info/rechtsstab/rechtsprechung/dokumentation/verfahre no2.html (Zugriff: 5.2.2007).

86 OLG Oldenburg 30.9.1991, NJW I992, 2438.

87 BVerfG 29.7.2004, NJW 2004, 2662 f. Vgl. auch: BVerfG 16.2.2006, NJW 2006, I 502 sowie Klose, Alexander, Forum Recht 2005 , I3 ff. 
Abmahnungen, im ersten Fall meist kostenlos durch die Anwaltskammer, kostenpflichtig durch den gegnerischen Rechtsanwalt. Spätestens nach der Mitteilung der Staatsanwaltschaft über die Einleitung eines Ordnungswidrigkeitenverfahrens (vgl. Art. I $\$ 8$ I Nr. I RBerG) stellen die meisten altruistischen Helfer ihre Tätigkeit ein.

Der RDGE erkennt an, dass eine ausreichende anwaltliche Versorgung mittelloser Personen durch die Rechtsanwaltschaft nicht immer sichergestellt ist. Bürgerschaftliches Engagement in Form von Rechtsberatung als Teil einer allgemeinen Lebenshilfe sei hier »objektiv notwendig «. ${ }^{88}$ Unentgeltliche Rechtsberatung durch karitative Organisationen und Vereinigungen soll daher erlaubt werden, vorausgesetzt, dass die Rechtsberatung zumindest unter Anleitung einer Person mit Befähigung zum Richteramt (also eines »Volljuristen«) erfolgt. Dies gilt auch für das bürgerschaftliche Engagement einzelner Bürger. Eine uneingeschränkte Erlaubnis zur unentgeltlichen Rechtsberatung gilt nur innerhalb familiärer, nachbarschaftlicher oder ähnlich enger persönlicher Beziehungen. Außerhalb dieses privaten Bereichs müssen auch Einzelpersonen zumindest die Anleitung durch einen Volljuristen nachweisen, werden jedoch die »erforderliche juristische Qualifikation regelmäßig in eigener Person besitzen« müssen. ${ }^{89}$

\section{c) Bedürfnis nach Rechtskommunikation}

Betroffen ist schließlich auch die Kommunikation über Recht. Zwar scheiterten die Abmahnversuche eines Rechtsanwalts gegen öffentlich-rechtliche und private Fernsehsender vor dem $\mathrm{BGH}, 9^{\circ}$ doch berichten auch Betreiber von Internet-Foren, in denen über Recht diskutiert und dabei mitunter konkrete Ratschläge erteilt werden, von einer anwaltlichen »Abmahnwelle«.

Dem steht im medizinischen Bereich ein breit gefächertes Angebot von Selbsthilfegruppen (vom »Deutschen Arthrose Forum « bis zum »Zuckerforum« für Diabetiker) gegenüber. Über das Internet, in Gruppentreffen und auf Kongressen tauschen Betroffene Erfahrungen über die Krankheit, Therapieformen und Ärzte aus. ${ }^{91}$ Möglich wird dies durch eine einschränkende Auslegung von $\mathbb{I}$ II HPG: Die »Ausübung der Heilkunde« setzt danach voraus, dass die Tätigkeit nach allgemeiner Auffassung besondere medizinische Kenntnisse erfordert, also nicht nur auf Allgemeinwissen beruht.92 Dagegen erfasste der zentrale Begriff der »Rechtsbesorgung « im RBerG »jede Tätigkeit, die auf eine unmittelbare Förderung konkreter fremder Rechtsangelegenheiten gerichtet ist, ohne Rücksicht auf deren Schwierigkeitsgrad «.93 Während der RDGE die »Freiheit der Rechtskommunikation 494 in den Medien insoweit klarstellt, als es sich um an die Allgemeinheit gerichtete Darstellungen und Erörterungen von Rechtsfragen und Rechtsfällen handelt, wird die Möglichkeit einer mit Bezug auf den konkre-

88 BR-Drs. 623/06, S. 79.

89 BR-Drs. 623/06, S. I 2 I.

90 BGH 6.I 2.200I, NJW 2002, 2877, 2879, 2880, 2882, 2884.

9I Eine Übersicht über die mehr als 400 Selbsthilfegruppen in diesem Bereich bietet www.selbsthilfeforum.de (Zugriff: 5.2.2007).

92 BGH 29.6.1987, NJW I987, 2928, 2929. Zur Tätigkeit eines „Wunderheilers“ vgl. BVerfG 3.6.2004, NJW 2004, 2890.

93 Rennen/Caliebe (Fn. 5I), Art. I $\int$ I Rn. 34.

94 Zur Rechtskommunikation: Detjen, Stephan: Referat auf dem 65. Deutschen Juristentag 2004, Abteilung Rechtsberatung, in: Verhandlungen des 65. Deutschen Juristentages Bonn 2004, Band II/I, München 2004 , S. R22. 
ten Einzelfall erfolgenden Weitergabe von Wissen und Erfahrungen von der Auslegung des neuen Begriffs der Rechtsdienstleistung abhängen.

\section{Summary:}

Am Beginn der Untersuchung stand die Frage nach dem Einfluss von Professionen auf die rechtliche Zulässigkeit nichtprofessioneller Tätigkeit. Es konnte gezeigt werden, dass neben einer regelmäßig schwer nachzuweisenden unmittelbaren Einflussnabme auf den Gesetzgeber vor allem ein mittelbarer Einfluss zu berücksichtigen ist. So müssen sich Tätigkeiten in dem von der Profession vermeintlich abgedeckten Bereich regelmäßig an deren Standards messen lassen. Der Hinweis auf Gefabren, die dem Verbraucher anderenfalls droben, erweist sich dabei als empirisch betrachtet äußerst vage. Gerade die Etablierung9s des aufgrund historischer Zufälligkeiten entstandenen Heilpraktikers ${ }^{96}$ stellt die umfassende Schutzbedürftigkeit des Verbrauchers in Frage. Dies gilt auch für die Bedürnisbefriedigung durch die Profession: Für das RBerG konnte gezeigt werden, dass sowohl im Bereich der entgeltlichen als auch der unentgeltlichen Tätigkeit unbefriedigte Bedürfnisse bestehen, denen nicht von vornherein jede Berechtigung abgesprochen werden kann. Der RDGE löst sich nur vorsichtig aus dem Schatten der Profession, indem er zwar einerseits daran festhält, dass es grundsätzlich Aufgabe der Anwaltschaft ist, Rechtsdienstleistungen zu erbringen, den Grundsatz aber dort einschränkt, wo die Profession nicht Willens oder in der Lage ist, diese Aufgabe vollständig zu erfüllen.

\section{Die neue Gesetzessammlung für die Politik Föderalismusreform bereits eingearbeitet}

NOMOSGESETZE

von Alemann I von Alemann

Staatsrecht für die Politik

Nomos
Staatsrecht für die Politik

Herausgegeben von Prof. Dr. Ulrich von Alemann, Universität Düsseldorf und Dr. Florian von Alemann, Berlin 2006, 378 S., brosch., 19,- $€$, ISBN 978-3-8329-2176-7

\footnotetext{
Bitte bestellen Sie bei Ihrer Buchhandlung

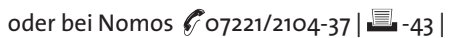
www.nomos.de | sabine.horn@nomos.de
}

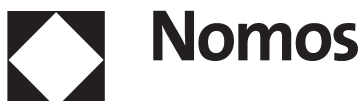

952004 wurden vom Statistischen Bundesamt ca. I 9.000 Heilpraktiker gezählt, denen 308.000 Ärzte gegenüber standen. Statistisches Bundesamt: Gesundheitspersonal 2005, Wiesbaden 2007. 96 Donhauser (Fn. 77), S. I 59. 